\title{
Evaluation of Two Storage Methods for Fungal Isolates of Fusarium sp. and Cochliobolus sativus
}

\author{
N. SAKR \\ Department of Agriculture, Atomic Energy Commission of Syria (AECS), Damascus, P.O. Box 6091, Syria
}

(Received: 29 October 2017; accepted: 24 January 2018)

\begin{abstract}
Comparison of two storage techniques (Castellani's method and freezing) was carried out for 16 isolates of four Fusarium head blight species and 54 isolates of Cochliobolus sativus causing spot blotch and common root rot. The viability, purity and morphological stability of cultures were verified for a one year period. Duplicate isolates were stored as a fungal suspension in sterile distilled water at $4{ }^{\circ} \mathrm{C}$, and fungal cultures by freezing at $-16^{\circ} \mathrm{C}$. The viability of 70 isolates was $100 \%$ for the two storage methods. Morphological alternations and pathogenic contaminations were not detected. All isolates were able to retain viability, purity and morphological stability by the two tested storage methods for over 12 months. Castellani's method and freezing were found to be safe, easy, inexpensive and reliable.
\end{abstract}

Keywords: Castellani’s method, freezing, viability.

The long-term preservation of plant pathogens in stable conditions without any morphological and physiological alternations is important for teaching, mycological and agricultural researches, and biotechnological purposes (Abd-Elsalam et al., 2010). Ideally, stored isolate should remain viable for extended period by lowering the metabolism until it reaches a stage of artificial dormancy (Guimaraes et al., 2014). This procedure prevents the accumulation of mutations causing morphological and biochemical alterations (Nakasone et al., 2004). Traditional techniques of the continuous sub-culturing are not suitable for maintaining a large number of fungal cultures. These techniques can lead to deleterious genetic and physiological changes and microbial contamination resulting in losses of virulence, aggressiveness or sporulation that take place as a consequence of long-term and frequent sub-culturing (Abd-Elsalam et al., 2010). Various maintenance methods for long periods of time have been evaluated with storage such as Castellani's method (Borman et al., 2006; Garcia-Garcia et al., 2014), silica gel (Windels et al., 1993; Arabi et al., 2007), sand (Windels et al., 1993; Arabi et al., 2007), freezing (Salustiano et al., 2008), cryopreservation (Homolka, 2013), and lyophilization (Milosevic et al., 2007), even though results are varied. No preservation method can be universally applied to all fungi and intraspecific variability makes it impossible to apply standard protocols, even at the species level (Ryan et al., 2000). The choice of a preservation technique for specific plant pathogens depends, among other factors, on the characteristics of the method, maintenance costs, importance of the collection and equipment availability (Guimaraes et al., 2014).

Although lyophilisation and cryopreservation are widely accepted to represent those methods of storage which best stabilized numerous fungal isolates for many years,

* Corresponding author; e-mail: ascientific@aec.org.sy 
such methods are not necessarily available in all mycology laboratories (Borman et al., 2006). Preservation of fungal cultures by the Castellani's method represents easy, simple, rapid and inexpensive technique. Also, isolates could be maintained depending on the species up to 20 years (Hornby and Bateman, 1998; Diogo et al., 2005; Elliott, 2005; Borman et al., 2006; Garcia-Garcia et al., 2014). This feasible method retained both viability and morphological, physiological and genetic stability upon the particular organism involved. Water preservation should be supplemented with other preservation methods to increase the chance of retaining both viability and morphological stability over long period (Borman et al., 2006).

Seventeen Fusarium species have been associated with Fusarium head blight. Spot blotch and common root rot diseases of barley are caused by Cochliobolus sativus. Fusarium head blight, spot blotch and common root rot are of the most damaging diseases of wheat and barley worldwide (Arabi et al., 2007; Imathiu et al., 2014; Kiseleva et al., 2016; Sakr, 2017, 2018). There is a paucity of reports about the storage of causal agents of Fusarium head blight and spot blotch diseases. For example, some Fusarium head blight species were maintained in viable conditions up to 10 years when preserved in soil, silica gel and lyophilization (Dhingra and Sinclair, 1985; Windels et al., 1993; Milosevic et al., 2007). Cultures of three spot blotch isolates retained viability and virulence during 2 years of storage in sand and silica gel, but a risk of mutation was found (Arabi et al., 2007). This study was initiated to evaluate the usefulness of two storage techniques (Castellani's method and freezing) in the case of 70 isolates of four Fusarium head blight species and two groups of $C$. sativus causing spot blotch and common root rot for a one year period. The viability, purity and morphological stability of the isolates were studied.

\section{Materials and Methods}

\section{Fungal isolates}

Sixteen Fusarium sp. isolates were recovered from wheat spikes showing Fusarium head blight symptoms in Ghab Plain in 2015, one of the principal Syrian wheat production areas. Fifty five $C$. sativus isolates were obtained in 2015 from both naturally infected barley leaves exhibiting spot blotch symptoms (32 isolates) and barley plants with common root rot symptoms (22 isolates) in different regions of Syria. (Table 1) gives identification for 70 isolates used in the current study. Survey data on wheat spikes with symptoms such as head blight and bleached grains on heads conducted across nine villages in Ghab Plain indicated that the main species were: F. culmorum, F. solani, F. verticillioides and $F$. equiseti. Although $F$. graminearum is considered the major causative of Fusarium head blight (Parry et al., 1995), this species was not found in the surveyed region. Isolates were grown separately in $9 \mathrm{~cm}$ Petri dishes containing potato-dextrose agar (PDA, HiMedia, HiMedia Laboratories) for 10 days at $22 \pm 1{ }^{\circ} \mathrm{C}$ in the dark to allow mycelial growth.

\section{Preservation by Castellani's method}

The fungal suspension composed of fungal spores and hyphae for 70 isolates was released by gently agitating $8 \mathrm{ml}$ of sterile distilled water on the surface of fungal cultures 
Table 1

Viability of 70 isolates for four Fusarium head blight species and two groups of Cochliobolus sativus causing spot blotch and common root rot preserved by Castellani's method and freezing for one year

\begin{tabular}{|c|c|c|c|c|c|}
\hline \multirow{2}{*}{$\begin{array}{l}\text { Fungal isolates } \\
\text { (identification) }\end{array}$} & \multicolumn{2}{|c|}{ Preservation methods } & \multirow{2}{*}{$\begin{array}{l}\text { Fungal isolates } \\
\text { (identification) }\end{array}$} & \multicolumn{2}{|c|}{ Preservation methods } \\
\hline & $\begin{array}{c}\text { Castellani's } \\
\text { method }\end{array}$ & freezing & & $\begin{array}{c}\text { Castellani's } \\
\text { method }\end{array}$ & freezing \\
\hline F1 (F. culmorum) & $+{ }^{a}$ & + & C.S. 59 (spot blotch) & + & + \\
\hline F2 (F. culmorum) & + & + & C.S. 17 (spot blotch) & + & + \\
\hline F3 (F. culmorum) & + & + & C.S. 34 (spot blotch) & + & + \\
\hline F28 (F. culmorum) & + & + & C.S. 21 (spot blotch) & + & + \\
\hline $\mathrm{F} 30$ (F. culmorum) & + & + & C.S. 89 (spot blotch) & + & + \\
\hline F7 (F. solani $)$ & + & + & C.S. 53 (spot blotch) & + & + \\
\hline F31 (F. solani) & + & + & C.S. 86 (spot blotch) & + & + \\
\hline $\mathrm{F} 35$ (F. solani $)$ & + & + & C.S. 74 (spot blotch) & + & + \\
\hline F20 (F. solani $)$ & + & + & C.S. 49 (spot blotch) & + & + \\
\hline F26 (F. solani $)$ & + & + & C.S. 9 (spot blotch) & + & + \\
\hline F29 (F. solani $)$ & + & + & C.S. 12 (spot blotch) & + & + \\
\hline F15 (F. verticillioides) & + & + & C.S. 63 (spot blotch) & + & + \\
\hline F16 (F. verticillioides) & + & + & C.S. 55 (spot blotch) & + & + \\
\hline F21 (F. verticillioides) & + & + & C.S. 41 (common root rot) & + & + \\
\hline F27 (F. verticillioides) & + & + & C.S. 50 (common root rot) & + & + \\
\hline F43 (F. equiseti) & + & + & C.S. 37 (common root rot) & + & + \\
\hline C.S. 14 (spot blotch) & + & + & C.S. 36 (common root rot) & + & + \\
\hline C.S. 27 (spot blotch) & + & + & C.S. 24 (common root rot) & + & + \\
\hline C.S. 32 (spot blotch) & + & + & C.S. 23 (common root rot) & + & + \\
\hline C.S. 92 (spot blotch) & + & + & C.S. 44 (common root rot) & + & + \\
\hline C.S. 20 (spot blotch) & + & + & C.S. 48 (common root rot) & + & + \\
\hline C.S. 2 (spot blotch) & + & + & C.S. 52 (common root rot) & + & + \\
\hline C.S. 80 (spot blotch) & + & + & C.S. 13 (common root rot) & + & + \\
\hline C.S. 7 (spot blotch) & + & + & C.S. 6 (common root rot) & + & + \\
\hline C.S. 18 (spot blotch) & + & + & C.S. 38 (common root rot) & + & + \\
\hline C.S. 30 (spot blotch) & + & + & C.S. 25 (common root rot) & + & + \\
\hline C.S. 93 (spot blotch) & + & + & C.S. 46 (common root rot) & + & + \\
\hline C.S. 16 (spot blotch) & + & + & C.S. 47 (common root rot) & + & + \\
\hline C.S. 87 (spot blotch) & + & + & C.S. 51 (common root rot) & + & + \\
\hline C.S. 83 (spot blotch) & + & + & C.S. 8 (common root rot) & + & + \\
\hline C.S. 45 (spot blotch) & + & + & C.S. 40 (common root rot) & + & + \\
\hline C.S. 11 (spot blotch) & + & + & C.S. 1 (common root rot) & + & + \\
\hline C.S. 9 (spot blotch) & + & + & C.S. 10 (common root rot) & + & + \\
\hline C.S. 15 (spot blotch) & + & + & C.S. 5 (common root rot) & + & + \\
\hline C.S. 26 (spot blotch) & + & + & C.S. 28 (common root rot) & + & + \\
\hline
\end{tabular}

\footnotetext{
a: +: mycelial growth.
} 
with suitable growth. It was aseptically taken and put in sterile glass ampoules which were hermetically closed and sealed with $2 \mathrm{~cm}$ Parafilm strips (Pechiny, Thomas Scientific) to prevent water evaporation and stored at $4{ }^{\circ} \mathrm{C}$.

For the viability test after a one year period, $300 \mu \mathrm{L}$ of fungal suspension were obtained with micropipette from sterile sealed glass ampoules for 70 isolates in an aseptic chamber. Fungal samples were seeded in Petri dishes with PDA and incubated under the conditions mentioned above to allow mycelial growth. If the fungal cultures grew, they were recorded as viable.

\section{Preservation by freezing}

PDA Petri dishes containing fungal cultures reaching suitable growth for 70 isolates were placed in the freezer at $-16^{\circ} \mathrm{C}$. After 12 months storage, frozen Petri dishes with PDA containing fungal cultures were thawed at $4{ }^{\circ} \mathrm{C}$ for 24 hours. Five agar plugs ( $5 \mathrm{~mm}$ diameter) of the colony were cut of for each isolate, then placed on the surface of Petri dishes with PDA and incubated under the conditions mentioned before to allow mycelial growth for viability test. Isolates exhibiting survival of at least one out of five agar plugs were considered viable.

For the two storage methods, viable cultures were examined for morphological observation (agreement of colony characteristics with the previously known identification) and contamination by bacteria or other fungi.

\section{Results and Discussion}

Recovered fungal isolates deposited in culture collection for prolonged periods can be used for taxonomic, genetic and agricultural purposes. The testing of more economical fungal storage techniques becomes necessary in small mycological laboratories in which the procedures of cryopreservation and lyophilization are not necessarily available (Abd-Elsalam et al., 2010). Bearing this in mind, viability of two storage methods (water at $4{ }^{\circ} \mathrm{C}$ and freezing at $-16{ }^{\circ} \mathrm{C}$ ) for 12 months were evaluated for causal agents of great economic importance diseases on wheat and barley. The storage key provided by Ryan et al. (2000) illustrates that storage in water and by freezing has been recommended for causal agents such as Fusarium head blight, spot blotch and common root rot due to the fact that these fungi readily produce asexual spores in culture and do not have motile spores.

In this study, each fungal isolate was considered viable if the fungal cultures did grow without microbial contamination at the same as that of the original culture and if the morphological criteria of the colony corresponded to the original description. Thus, this assessment method depends on visual evaluation for the viability, purity and morphological stability. The evaluation method employed in the current research has been applied in several studies for human and phytopathogenic agents and yielded satisfactory results for extended storage period: 147 species belonging to 66 genera of filamentous fungi, yeasts, and aerobic actinomycetes (McGinnis et al., 1974), 160 medical species represented 594 isolates (de Capriles et al., 1989), 1474 clinical and environmental isolates of molds, yeasts, aerobic actinomycetes, and algae belonging to 164 genera (382 taxa) (Pasarell and McGinnis, 1992), 18 strawberry pathogenic fungi of genera Colletotrichum 
and Phomopsis (Legard and Chandler, 2000), 43 medical species of genera Aspergillus, Cladosporium, Fusarium, Mucor, Penicillium and Rhizopus (Diogo et al., 2005), 179 isolates of 45 species of pathogenic filamentous fungi (Borman et al., 2006), and 12 potentially toxigenic species of the genera Aspergillus and Penicillium (Guimaraes et al., 2014).

(Table 1) showed that the viability of 70 isolates of five species was $100 \%$ for the two storage methods for a period of one year. Growth was assessed visually (Fig. 1), viability was determined by growing mycelial culture in Petri dishes with PDA. For 12 months, duplicate 16 out of 70 isolates were tested monthly for water storage and in three months intervals for freezing. 100\% viability of cultures was recovered of 16 isolates from two storage methods at each time point (data not shown). Our results are comparable with these reviewed by Abd-Elsalam et al. (2010), they noted that storage methods incorporating fungal spores and mycelium had high viability rates. No morphological alternation and contamination by bacteria or other fungi which harm the preservation of fungal colonies were detected in 70 isolates by the two storage methods (Fig. 1). In addition, Elliott (2005) found that water storage did not appear to affect clear morphological colony aspects of Gaeumannomyces graminis var. graminis. Moreover, no visible morphological changes were observed in strawberry pathogenic fungi by freezing at $-95^{\circ} \mathrm{C}$ (Legard and Chandler, 2000).
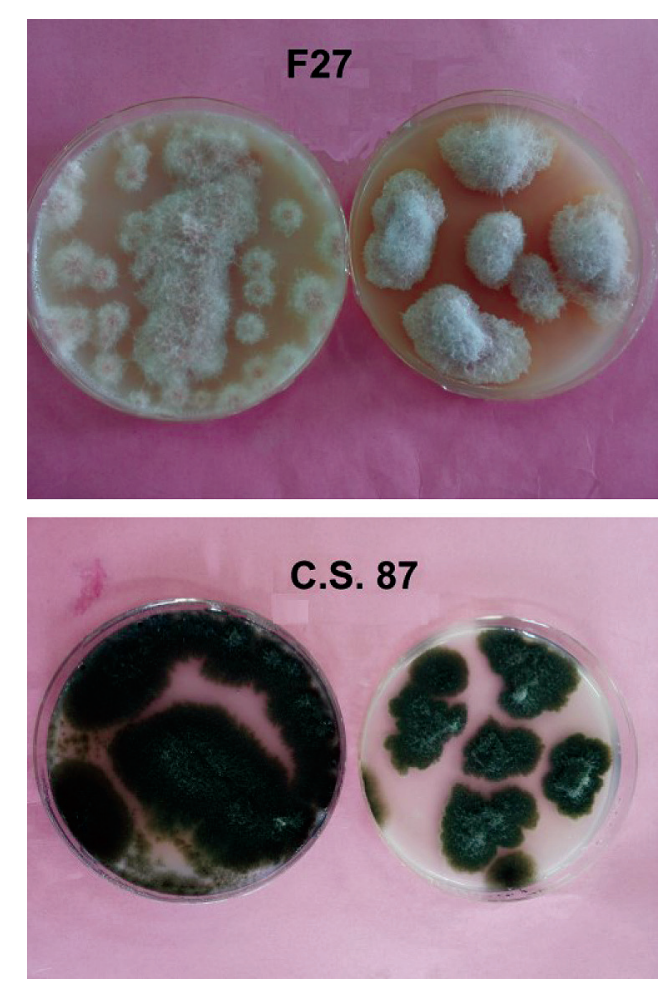

Fig. 1. Fungal cultures of two isolates F27 (Fusarium verticillioides)

and C.S. 87 (Cochliobolus sativus) on Petri dish with potato-dextrose agar obtained from cultures preserved by Castellani's method (left side) and freezing (right side) for one year 
For preservation of cultures by Castellani's method, the selection of good sporulating cultures and sufficient suspension consisting of spores and mycelia were the most important factors influencing survival of fungi in water over a longer period of time (McGinnis et al., 1974). The volume of water for preparation of the fungal suspension in this experiment was suggested by McGinnis et al. (1974) who observed that this suspension can enhance the capacity of the fungus to survive for up 60 months. In the current research, too much inoculum for the volume of water may jeopardize the ability of the fungus to withstand long-term storage (data not shown). Immersion of fungal inoculum consisting of spores and fragments of hyphae into ampoules containing sterile water yielded viability rates of $100 \%$ with Fusarium head blight, spot blotch and common root rot pathogens (Table 1). The culture remains in an inactive state as water suppresses the growth of the fungi and the culture gets revived when transferred to fresh medium besides avoiding morphological changes in most fungi (Nakasone et al., 2004). High recovery rates were reported for human and phytopathogenic cultures stored in sterile water (McGinnis et al., 1974; de Capriles et al., 1989; Hornby and Bateman, 1998; Diogo et al., 2005; Elliott, 2005; Borman et al., 2006; Garcia-Garcia et al., 2014; Guimaraes et al., 2014).

Although most metabolic activity is suspended below $-70{ }^{\circ} \mathrm{C}$, ice recrystallization and other biophysical processes that can affect cell survival may still be active at temperatures above $-130{ }^{\circ} \mathrm{C}$ (Legard and Chandler, 2000). Changes in the morphology of hyphae during freezing and thawing, and variable viability following thawing, necessitate the application of different freezing and thawing rates (Morris et al., 1988). This requires species-specific information to be considered. The survival of the cultures stored and thawed with and without freezing and thawing modules was excellent during our investigation. Our results are in accordance with data found by Legard and Chandler (2000). In our study, frozen PDA Petri dishes containing fungal cultures were used once due to the multiple freeze-thaw cycles of frozen fungal cultures could have caused genetic damage (Legard and Chandler, 2000). Arabi et al. (2007) found that the viability of spot blotch isolates was not best maintained for 2 years by using frozen conidia at $-20^{\circ} \mathrm{C}$. Larger fungal spores (such those for Fusarium sp. and C. sativus used in this study) tend to collapse during freezing $-20^{\circ} \mathrm{C}$, and the damage is not reversible by hydration (Arabi et al., 2007). Due to these possible difficulties, freezing processes were regulated at $-16^{\circ} \mathrm{C}$ in a freezer available in our small mycological laboratory.

\section{Conclusion}

Results in the current study highlighted the possibility of storage for 70 isolates of five species in a stable condition without any morphological alternations by Castellani's method and freezing for 1-year. Castellani's method and freezing appear to be easy, convenient, economical, and effective tools for preservation Fusarium head blight, spot blotch and common root rot pathogens for up to 12 months. Viability tests will be necessary in F. graminearum, if isolated, to verify Árpád Szécsi's note (information provided by the Reviewer) in which this fungus cannot be preserved under sterile distilled water at $4{ }^{\circ} \mathrm{C}$. The revival techniques in the case of Castellani's method and freezing at $-16^{\circ} \mathrm{C}$ are less messy than that of FHB and SB cultures stored with lyophilization, sand or silica gel. 
More studies for longer periods of time are in progress due to the tested times correspond to a period of short duration (12 months).

\section{Acknowledgements}

The author would like to thank the Director General of Atomic Energy Commission of Syria and the Head of the Agriculture Department for continuous support throughout this work.

\section{Literature}

Abd-Elsalam, K. A., Yassin, M. A., Moslem, M. A., Bahkali, A. H., McKenzie, E. H. C., Stephenson, S. L., Cai, L. and Hyde, K. D. (2010): Culture collections, the new herbaria for fungal pathogens. Fungal Divers. $45,21-32$.

Arabi, M. I. E., Jawhar, M. and Al-Daoude, A. (2007): Viability of Cochliobolus sativus cultures after storage under different conditions. J. Plant Pathol. 89, 79-83.

Borman, A. M., Szekely, A., Campbell, C. K. and Johnson, E. M. (2006): Evaluation of the viability of pathogenic filamentous fungi after prolonged storage in sterile water and review of recent published studies on storage methods. Mycopathologia 161, 361-368.

De Capriles, C. H., Mata, S. and Middelveen, M. (1989): Preservation of fungi in water (Castellani): 20 years. Mycopathologia 106, 73-79.

Dhingra, O. D. and Sinclair, J. B. (1985): Basic Plant Pathology Methods. CRC Press, Florida, pp. 1-355.

Diogo, H. C., Sarpieri, A. and Pires, M. C. (2005): Fungi preservation in distilled water. An. Bras. Dermatol. 80, 591-594.

Elliott, M. L. (2005): Survival, growth and pathogenicity of Gaeumannomyces graminis var. graminis with different methods of long term storage. Mycologia 97, 901-907.

Garcia-Garcia, M., Rocha-Zavaleta, L., Valdez-Cruz, N. A., Mauricio, A. and Trujillo-Roldan, M. A. (2014): Conservation of the mycelia of the medicinal mushroom Humphreya coffeata (Berk.) Stey. in sterile distilled water. Methods X 1, 19-22.

Guimaraes, L. C., Fernandes, A. P., Chalfoun, S. M. and Batista, L. R. (2014): Methods to preserve potentially toxigenic fungi. Braz. J. Microbiol. 4, 43-47.

Homolka, L. (2013): Methods of cryopreservation in fungi. In: V. K. Gupta et al. (eds): Laboratory Protocols in Fungal Biology: Current Methods in Fungal Biology, Springer, New York, pp. 9-16.

Hornby, D. and Bateman, G. L. (1998): Take-all Disease of Cereals: A Regional Perspective. CAB International, Wallingford, pp. 1-384.

Imathiu, S. M., Edwards, S. G., Ray, R. V. and Back, M. (2014): Artificial inoculum and inoculation techniques commonly used in the investigation of Fusarium head blight in cereals. Acta Phytopathol. et Entomol. Hung. 49, 129-139.

Kiseleva, M. I., Ovsyankina, A. V., Kolomiets, T. M., Zhemchuzhina, N. S. and Glinushkin, A. P. (2016): Some aspects of the distribution of Fusarium on cereals of Russia. Acta Phytopathol. et Entomol. Hung. 51, $183-191$.

Legard, D. E. and Chandler, C. K. (2000): Cryopreservation of strawberry pathogen in mechanical ultra-low temperature freezer. Hort Science 35, 1357.

McGinnis, M. R., Padhye, A. A., and Ajello, L. (1974): Storage of stock cultures of filamentous fungi, yeasts, and some aerobic Actinomycetes in sterile distilled water. Appl. Microbiol. 28, 218-222.

Milosevic, M. B., Medic-Pap, S. S., Ignjatov, M. V. and Petrovic, D. N. (2007): Lyophilization as a method for pathogens long term preservation. Proc. Nat. Sci. Matica. Srpska. Novi. Sad. 113, 203-210.

Morris, G. J., Smith, D. and Coulson, G. E. (1988): A comparative study of the morphology of hyphae during freezing with the viability upon thawing of 20 species of fungi. J. Gen. Microbiol. 134, 2897-2906.

Nakasone, K. K., Peterson, S. W. and Jong, S. C. (2004): Preservation and distribution of fungal cultures. In: G. M. Mueller et al. (eds): Biodiversity of Fungi: Inventory and Monitoring Methods. Elsevier Academic Press, San Diego, pp. 37-47. 
Parry, D. W., Jekinson, P. and MCleod, L. (1995): Fusarium ear blight (scab) in small grain cereals-a review. Plant Pathol. 44, 207-238.

Pasarell, L. and McGinnis, M. R. (1992): Viability of fungal cultures maintained at $-70{ }^{\circ} \mathrm{C}$. J. Clin. Microbiol. 30, 1000-1004.

Ryan, M. J., Smith D. and Jeffries P. (2000): A decision-based key to determine the most appropriate protocol for the preservation of fungi. World J. Microbiol. Biotechnol. 16, 183-186.

Sakr, N. (2017): In vitro assessment of Fusarium head blight spp. on wheat cultivars. Arch. Phytopathol. Plant Protect. 50, 254-261.

Sakr, N. (2018): Aggressiveness variation among and within Fusarium head blight species on barley in vitro. Acta Phytopathol. et Entomol. Hung. DOI: 10.1556/038.52.2017.033.

Salustiano, M. E., Pozza, E. A., Ferraz, F. A. C. and Castro, H. A. (2008): Viability of Puccinia pisidii urediniospores stored in different environments. Trop. Plant Pathol. 33, 313-316.

Windels, C. E., Burnes, P. M. and Kommedahll, T. (1993): Fusarium species stored on silica gel and soil for ten years. Mycologia 85, 21-23. 